\title{
Resistencia metabólica a insecticidas organofosforados en Anopheles aquasalis Curry 1932, municipio Libertador, estado Sucre, Venezuela
}

\author{
Darjaniva Molina, Luisa Elena Figueroa
}

Instituto de Altos Estudios en Salud Pública "Dr. Arnoldo Gabaldón”, Centro de Estudios de Enfermedades Endémicas y Salud Ambiental, Ministerio del Poder Popular para la Salud, Maracay, Aragua, República Bolivariana de Venezuela

Introducción. Se realizó un estudio centrado en las localidades de Catuaro, Guayana, Platanito y Río de Agua, del municipio Libertador, estado Sucre, Venezuela, región con transmisión de malaria, donde el vector principal es Anopheles aquasalis.

Objetivo. Evaluar la expresión de resistencia a los insecticidas organofosforados fenitrotión y metilpirimifos, usados para el control de An. aquasalis.

Materiales y métodos. En mosquitos adultos de las localidades mencionadas, se realizaron pruebas biológicas y se identificaron los mecanismos de resistencia in vitro por medio de pruebas bioquímicas.

Resultados. Se detectó elevación de las esterasas alfa y beta, y alteración de la acetilcolinesterasa. Las enzimas oxidasas de función múltiple en las poblaciones de An. aquasalis de tres de las localidades evaluadas, también resultaron alteradas, por lo que ambos sistemas enzimáticos pudieran estar interviniendo en la expresión de resistencia a insecticidas organofosforados en las poblaciones de estudio. Por otro lado, la actividad enzimática de la glutatión-S-transferasa sólo está operando en Río de Agua.

Conclusión. Los datos obtenidos aportan información para un mejor conocimiento de la resistencia a insecticidas en esta especie de importancia médica, con el fin de implementar la rotación de insecticidas como estrategia dentro de un programa de manejo integrado.

Palabras clave: Anopheles, insecticidas organofosforados, acetilcolinesterasa, esterasas, oxidorreductasa, resistencia a los insecticidas.

Metabolic resistance to organophosphate insecticides in Anopheles aquasalis Curry 1932, Libertador municipality, Sucre State, Venezuela

Introduction. A study of insecticide resistance was undertaken at focal level in the localities Catuaro, Guayana, Platanito and Rio de Agua, Libertador County, Sucre State, Venezuela, a region with malaria transmission, where Anopheles aquasalis is the main vector.

Objective. Insecticide resistance was assessed in the organophosphate insecticides fenitrothion and pirimiphos methyl, both of which are used in the control of Anopheles aquasalis.

Materials and methods. In adult mosquitoes, biological tests were performed and identification of resistance mechanisms in vitro by biochemical tests.

Results. Elevated levels of alpha and beta esterases were detected, as well as altered acetylcholinesterase activity. Multifunction oxidase enzymes in populations of Anopheles aquasalis in three of the locations evaluated were also altered; therefore, both enzyme systems may be involved in the expression of resistance to organophosphate insecticides in the study populations. The enzyme activity of glutathione-S-transferase was noted only in Rio de Agua.

Conclusions. A better understanding of the resistance to insecticides was obtained in this species of medical importance. These findings will assist the implementation the practice of insecticide rotation as a strategy within an integrated management program.

Key words: Anopheles; insecticides, organophosphate; acetylcholinesterase, esterases, oxidoreductases, insecticide resistance. 
El paludismo, o malaria, es una enfermedad parasitaria que se transmite de un humano a otro por la picadura de mosquitos del género Anopheles Ross 1897 infectados con parásitos protozoarios del género Plasmodium Laverán 1880. Esta enfermedad constituye un problema de salud en gran parte de los países tropicales y subtropicales. Los Centers for Disease Control and Prevention (CDC) de los Estados Unidos calculan que cada año se presentan de 300 a 500 millones de casos de malaria y que más de un millón son mortales. En algunas regiones del mundo, el mosquito que transmite la malaria ha desarrollado resistencia ante sustancias insecticidas, mientras que el parásito ha desarrollado resistencia a los antipalúdicos. Esto ha dificultado el control tanto de la tasa de infección como de la diseminación de la enfermedad (1).

Los insecticidas han jugado un papel importante en el control de insectos vectores de enfermedades desde principios del siglo XX. La Organización Mundial de la Salud (OMS) está promoviendo el control integrado de vectores, incluyendo medidas alternativas como el control biológico o el manejo ambiental, en el tiempo y lugar donde ellas sean efectivas y aplicables; también promueve el uso de insecticidas cuando el control biológico no es una alternativa. En tal sentido, los insecticidas continúan siendo un elemento vital en programas de control. Sin embargo, existe una importante habilidad de las poblaciones de insectos para desarrollar resistencia a varias clases de insecticidas que se han usados (2).

La resistencia ha sido un problema en muchos grupos de insectos vectores de enfermedades emergentes. Aunque los mecanismos por los cuales los insecticidas llegan a ser menos

\footnotetext{
Correspondencia:

Darjaniva Molina, Instituto de Altos Estudios en Salud Pública "Dr. Arnoldo Gabaldon", Avenida Bermúdez Sur No. 93, Maracay, estado Aragua, República Bolivariana de Venezuela.

Teléfono: (0058-0243) 241 2846; fax: (0058-0243) 2336933 darja2410@gmail.com

Recibido:05/03/09; aceptado:16/06/09
}

efectivos son similares en todas las especies de vectores, cada problema de resistencia es potencialmente único y puede involucrar patrones complejos de resistencia focal (3).

El dicloro-difenil-tricloroetano (DDT) fue el primer insecticida empleado en el control de los mosquitos y en 1947 se reportó el primer caso de resistencia al DDT en el género Aedes. Después de esto se ha encontrado resistencia en más de 100 especies de mosquitos a uno o más insecticidas y más de 50 pertenecen al género Anopheles. Los insecticidas empleados para el control de la malaria incluyen los grupos de los organofosforados, carbamatos y piretroides (4).

Se conocen varios mecanismos de resistencia bioquímica, los cuales incluyen: aumento del metabolismo de los plaguicidas a productos no tóxicos, incremento de la sensibilidad del sitio blanco, disminución en las tasas de penetración de los insecticidas y aumento de las tasas de excreción de los insecticidas (5). Se ha demostrado que muchos sistemas enzimáticos están involucrados en la desintoxicación de las cuatro principales clases de insecticidas $y$, usualmente, la resistencia se atribuye al incremento en las actividades enzimáticas del vector. La elevación de los niveles de glutatión-Stransferasa, monooxigenasas, carboxilesterasas y acetilcolinesterasa insensible, indica tolerancia y resistencia a las principales clases de insecticidas que han sido desarrollados y usados contra artrópodos de importancia agrícola y en salud pública (6).

Particularmente en Venezuela, el uso prolongado de insecticidas ha contribuido a la aparición de resistencia en poblaciones de $A n$. aquasalis, vector principal de malaria en el estado Sucre, lo cual ha dificultado el control de esta enfermedad por medio de la eliminación del vector (7). Para 1997, An. aquasalis se reportó como sensible a insecticidas organofosforados, entre los que se incluye metil-pirimifos; no obstante, este insecticida fue el que presentó mayor valor de tiempo letal, en relación con los insecticidas malatión, fentión y fenitrotión, lo cual evidencia que ya se estaba desarrollando resistencia incipiente a metil-pirimifos. 
Por otro lado, el programa de control de la malaria en el estado Sucre está basado en la aplicación espacial de insecticidas organofosforados contra mosquitos adultos y la administración masiva de medicamentos antipalúdicos; infortunadamente, en la actualidad existe resistencia tanto a fármacos como a insecticidas convencionales (8). En la historia particular del uso de insecticidas en salud pública para el control de malaria, los datos señalan que el uso intensivo de malatión fue de $94 \%$ y de $50 \%$ de fenotión durante los años 1998 y 1999 cuando la casuística era de 4.674 casos. El actellic (metil-pirimifos al 50\%) fue incorporado desde el año 2000, con 5.011 casos de malaria en el estado Sucre, hasta el 2005, de manera ininterrumpida (9).

\section{Materiales y métodos}

\section{Insectos}

La muestra estuvo comprendida por la fauna de anofeles del municipio Libertador en el estado Sucre. Se evaluaron mosquitos adultos de $A n$. aquasalis de las localidades de Catuaro (10⒊'

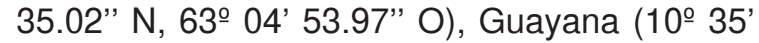

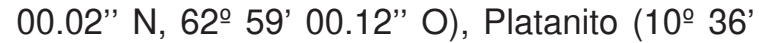

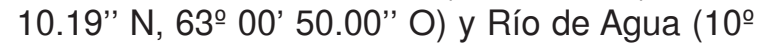
34' 37.93"' N, 62 59' 46.70" O), recolectados en el peridomicilio entre las 18:00 y las 20:00, en cuatro establos para ganado vacuno, uno en cada localidad; se hizo sobre cebo animal y reposando en vegetación, con una frecuencia de siete días por mes, de mayo a octubre, durante la estación lluviosa en los años 2006 y 2007. Cabe destacar que, en el estado Sucre, los picos de mayor densidad de An. aquasalis se presentan generalmente en los meses de lluvia $(10,11)$.

\section{Insecticidas}

Se evaluaron dos insecticidas organofosforados, fenitrotión (95\% P/P) y metil-pirimifos $(89,8 \%$ $\mathrm{P} / \mathrm{P})$, en presentación grado técnico, sin valor comercial, que fueron suministrados por Insecticidas Internacionales Compañía Anónima, INICA.

\section{Pruebas biológicas}

Los bioensayos se realizaron siguiendo el método de las botellas de los CDC. Los mosquitos adultos hembra recolectados se expusieron a botellas de vidrio tipo Wheaton de $250 \mathrm{ml}$, tratadas con soluciones cetónicas de insecticidas, las cuales se usaron como cámaras de prueba para detectar la resistencia a los insecticidas en mosquitos adultos.

En ensayos anteriores se definió la dosis diagnóstica como la menor dosis que mata el mayor porcentaje de los insectos expuestos y se usó el límite de $\geq 60$ minutos para categorizar las poblaciones como resistentes o sensibles. Por ello, se evaluaron los insecticidas fenitrotión y metil-pirimifos, a la concentración de $5 \mu \mathrm{g} / \mathrm{ml}$. Las botellas de control del experimento sólo fueron tratadas con acetona.

Los ensayos se realizaron a temperaturas aproximadas de $26^{\circ} \mathrm{C} \pm 2^{\circ} \mathrm{C}$ y humedad relativa de $75 \% \pm 5 \%$. Se expusieron, aproximadamente, 10 mosquitos adultos hembras por botella y se evaluaron cuatro repeticiones por cada concentración de insecticida y dos repeticiones como grupo control. Cada ensayo se hizo por triplicado y se empleó un promedio de 120 mosquitos por bioensayo en cada una de las localidades, para cada insecticida. Los ensayos fueron replicados tres veces en días distintos, durante el tiempo que se lograba el $100 \%$ de mortalidad. De esta manera se determinó el efecto del insecticida en función del tiempo de exposición (12,13).

\section{Pruebas bioquímicas}

Cada mosquito fue triturado en $50 \mu \mathrm{l}$ de solución tampón de fosfato y diluido a $0,5 \mathrm{ml}$ de la misma solución. Se tomaron alícuotas de $50 \mu \mathrm{l}$ de cada muestra y se colocaron en placas para microtitulación de 96 pocillos. Se evaluaron seis diferentes enzimas que confieren resistencia a insecticidas; esterasas a y $\beta$, oxidasas de función múltiple (OFM), glutatión-S-tranferasa (GST), acetilcolinesterasa (AChe) y acetilcolinesterasa insensible (AChei). Los substratos utilizados en cada ensayo incluyeron: a y $\beta$-naftil-acetato para las esterasas no específicas; glutatión reducido y CDNB (1-chloro-2,4'-dinitrobenzeno) para la GST; TMBZ (3,3`,5,5`-tetrametil benzidina) para las oxidasas (hemoperoxidasa); el yoduro de acetiltiocolina se usó para medir la actividad 
de la acetilcolinesterasa normal, y para la acetilcolinesterasa insensible se añadió el insecticida carbamato propoxur a la muestra.

La absorbancia se midióen un lector de ELISA, Multiskan Plus $\AA^{\circledR}$ de Fisher Scientific, empleando filtros de $405 \mathrm{~nm}$ para GST, AChe y AChei; para las esterasas y oxidasas se empleó filtro de 620 nm (14-17).

Los resultados obtenidos para las pruebas, se analizaron en función de los valores de absorbancia, según el siguiente criterio: para las esterasas $\alpha$ y $\beta(>0,8)$, oxidasa de función múltiple $(>0,5)$ y las glutatión-S-transferasa $(>0,2)$. La actividad de la acetilcolinestarasa fue clasificada como "no alterada", "incipientemente alterada" o "alterada", cuando el porcentaje de inhibición era menor de $15 \%$, entre $15 \%$ y $50 \%$ o mayor de $50 \%$, respectivamente $(18,19)$.

A los datos obtenidos se les aplicó análisis de varianza de una sola vía no paramétrico de Kruskal Wallis y prueba de medias de Bonferroni a un nivel de significación del 0,05\%. El análisis aplicado es del tipo en que se establece comparación en el conjunto de las cuatro localidades experimentales, para cada mecanismo, por lo que se estableció un único valor de $\mathrm{P}$ y $\mathrm{F}$ para cada comparación.

\section{Resultados}

En la figura 1 se presenta la relación tiempo (dosis) mortalidad de An. aquasalis al fenitrotión, y se registra que los mosquitos de las cuatro localidades: Catuaro, Río de Agua, Platanito y Guayana, resultaron resistentes y se observó $100 \%$ de mortalidad en $60,70,120$ y 150 minutos, respectivamente. Así, también, en la figura 2 se presenta la relación tiempo (dosis) mortalidad de $A n$. aquasalis al metil-pirimifos; se encontró que los mosquitos de todas las localidades resultaron resistentes y se bservó $100 \%$ de mortalidad en $90,60,70$ y 80 minutos, respectivamente.

Los resultados de la medición de los niveles de esterasas alfa y beta de poblaciones de An. aquasalis de las localidades evaluadas, se muestran en las figuras 3a-3d. Los valores de absorbancia oscilaron entre 0,8 y 3,7, y mantienen una distribución normal.

En el cuadro 1 se muestra el ANOVA de una sola vía para esterasas alfa, el cual reveló similitud entre Catuaro y Guayana, y diferencias significativas $(p<0,0001)$ de éstas con Platanito y Río de Agua $(F=226,42)$. Para esterasas beta, se encontraron diferencias significativas $(p<0,0001)$ entre las poblaciones de mosquitos

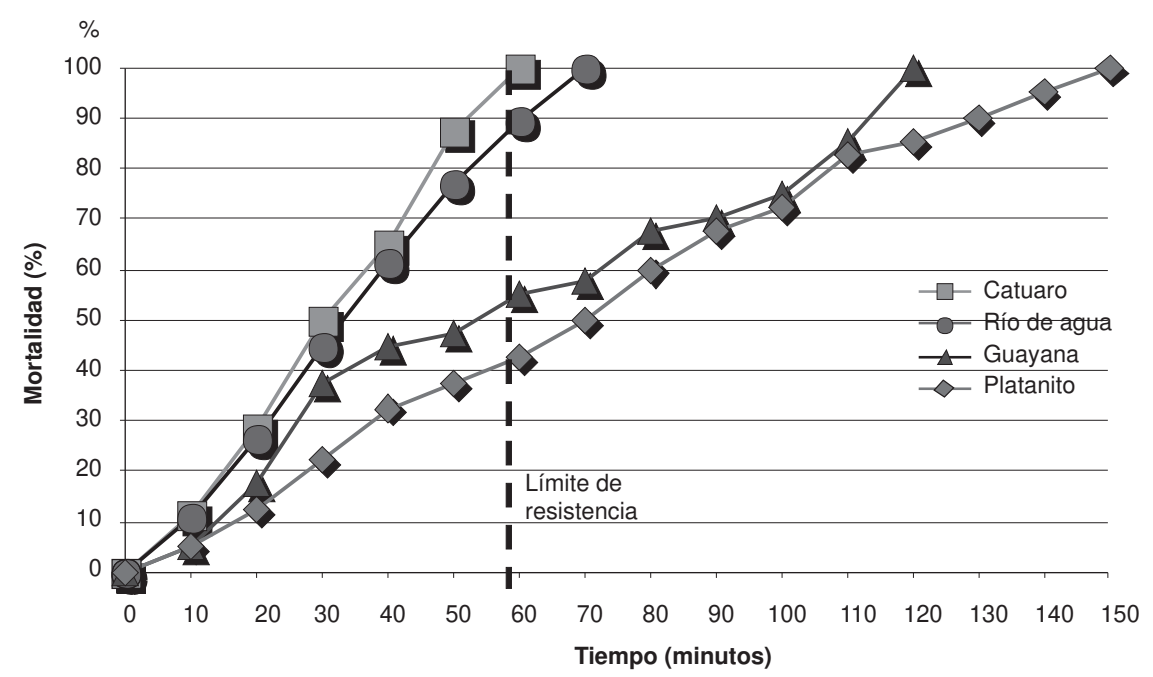

Figura 1. Tendencia de los datos tiempo-mortalidad en adultos de Anopheles aquasalis expuestos al organofosforado fenitrotión. Municipio Libertador, estado Sucre 


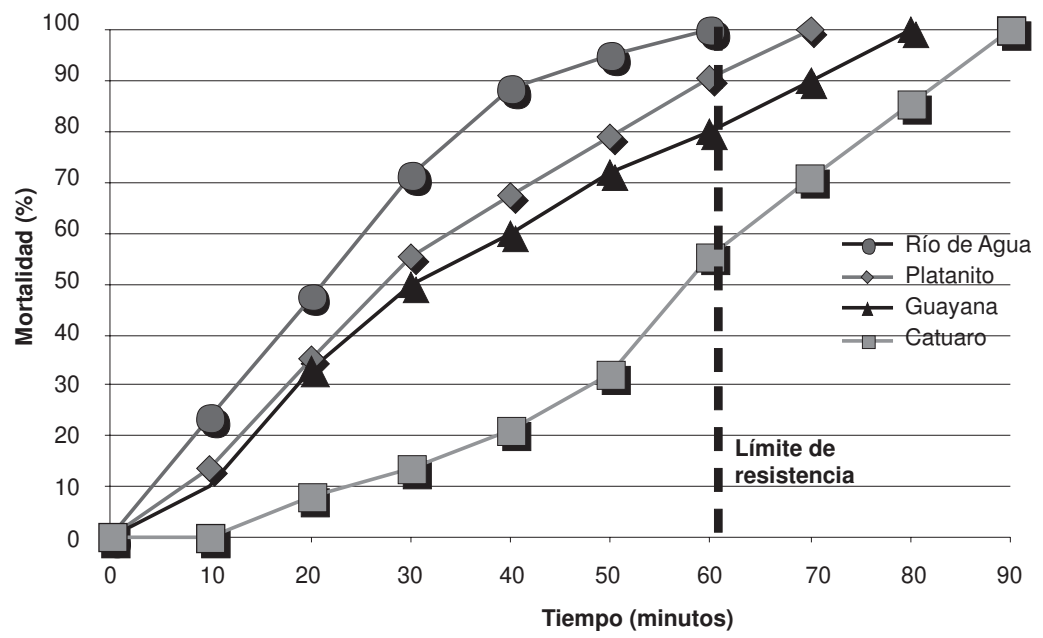

Figura 2. Tendencia de los datos tiempo-mortalidad en adultos de Anopheles aquasalis expuestos al organofosforado pirimifosmetil, municipio Libertador, estado Sucre

Cuadro 1. Estadística descriptiva y análisis de varianza por una sola vía, de la actividad enzimática en adultos de Anopheles aquasalis medidos en absorbancia, municipio Libertador, estado Sucre, Venezuela.

\begin{tabular}{|c|c|c|c|c|c|c|}
\hline Mecanismo & Localidad & $\mathbf{N}$ & Media & $\begin{array}{c}\text { Desviación } \\
\text { estándar }\end{array}$ & $\begin{array}{c}F^{*} \\
\text { (ANOVA) }\end{array}$ & $\mathbf{P}^{*}$ \\
\hline ATCH no inhibida & $\begin{array}{l}\text { Catuaro } \\
\text { Guayana } \\
\text { Platanito } \\
\text { Río de Agua }\end{array}$ & $\begin{array}{l}84 \\
90 \\
90 \\
90\end{array}$ & $\begin{array}{l}0,6473 \\
0,3154 \\
0,7584 \\
0,3045\end{array}$ & $\begin{array}{l}0,0247 \\
0,0113 \\
0,0409 \\
0,0142\end{array}$ & 82,36 & $<0,0001$ \\
\hline ATCH inhibida & $\begin{array}{l}\text { Catuaro } \\
\text { Guayana } \\
\text { Platanito } \\
\text { Río de Agua }\end{array}$ & $\begin{array}{l}84 \\
90 \\
90 \\
90\end{array}$ & $\begin{array}{l}0,18642 \\
0,31553 \\
0,21164 \\
0,27270\end{array}$ & $\begin{array}{l}0,00418 \\
0,00596 \\
0,00908 \\
0,00933\end{array}$ & 59,72 & $<0,0001$ \\
\hline EST alfa & $\begin{array}{l}\text { Catuaro } \\
\text { Guayana } \\
\text { Platanito } \\
\text { Río de Agua }\end{array}$ & $\begin{array}{l}84 \\
90 \\
90 \\
90\end{array}$ & $\begin{array}{l}2,7074 \\
3,0957 \\
1,8696 \\
2,3201\end{array}$ & $\begin{array}{l}0,1922 \\
0,0200 \\
0,0241 \\
0,0584\end{array}$ & 226,42 & $<0,0001$ \\
\hline EST beta & $\begin{array}{l}\text { Catuaro } \\
\text { Guayana } \\
\text { Platanito } \\
\text { Río de Agua }\end{array}$ & $\begin{array}{l}84 \\
90 \\
90 \\
90\end{array}$ & $\begin{array}{l}2,1202 \\
2,1778 \\
1,9319 \\
1,8694\end{array}$ & $\begin{array}{l}0,0157 \\
0,00803 \\
0,0200 \\
0,0480\end{array}$ & 28,44 & $<0,0001$ \\
\hline MFO & $\begin{array}{l}\text { Catuaro } \\
\text { Guayana } \\
\text { Platanito } \\
\text { Rio de Agua }\end{array}$ & $\begin{array}{l}84 \\
90 \\
90 \\
90\end{array}$ & $\begin{array}{l}1,1842 \\
1,3470 \\
0,8123 \\
0,3269\end{array}$ & $\begin{array}{l}0,0316 \\
0,0299 \\
0,0552 \\
0,0197\end{array}$ & 154,27 & $<0,0001$ \\
\hline GST & $\begin{array}{l}\text { Catuaro } \\
\text { Guayana } \\
\text { Platanito } \\
\text { Río de Agua }\end{array}$ & $\begin{array}{l}84 \\
90 \\
90 \\
90\end{array}$ & $\begin{array}{l}0,21090 \\
0,17440 \\
0,17546 \\
0,24736\end{array}$ & $\begin{array}{l}0,00408 \\
0,00538 \\
0,00417 \\
0,00215\end{array}$ & 72,14 & $<0,0001$ \\
\hline
\end{tabular}

$\mathrm{N}=$ individuos evaluados

* Por cada mecanismo y para todas las localidades 


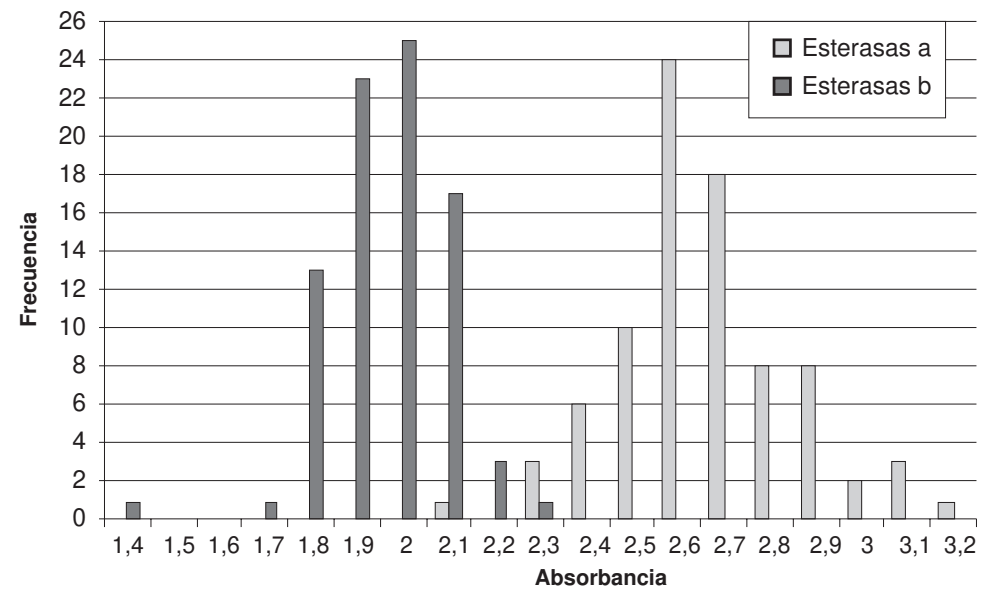

Figura 3a. Niveles de esterasas en adultos de Anopheles aquasalis de Catuaro, municipio Libertador, estado Sucre.

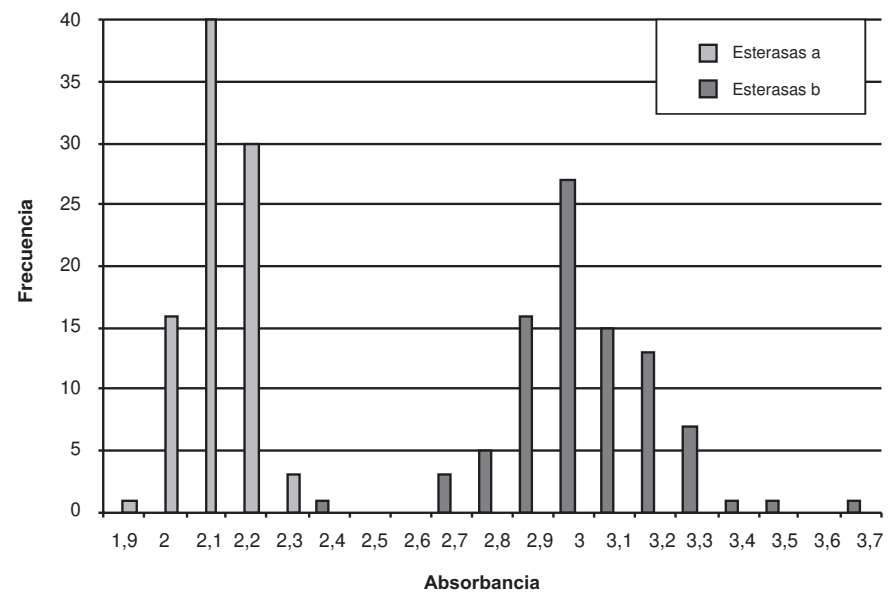

Figura 3b. Niveles de esterasas en adultos Anopheles aquasalis de Guayana, municipio Libertador, estado Sucre.

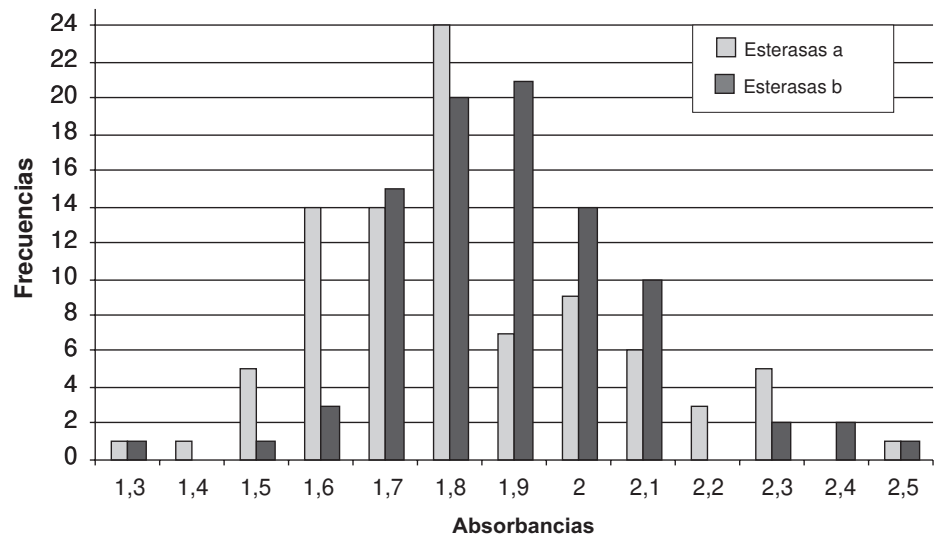

Figura 3c. Niveles de esterasas de adultos Anopheles aquasalis de Platanito, municipio Libertador, estado Sucre. 


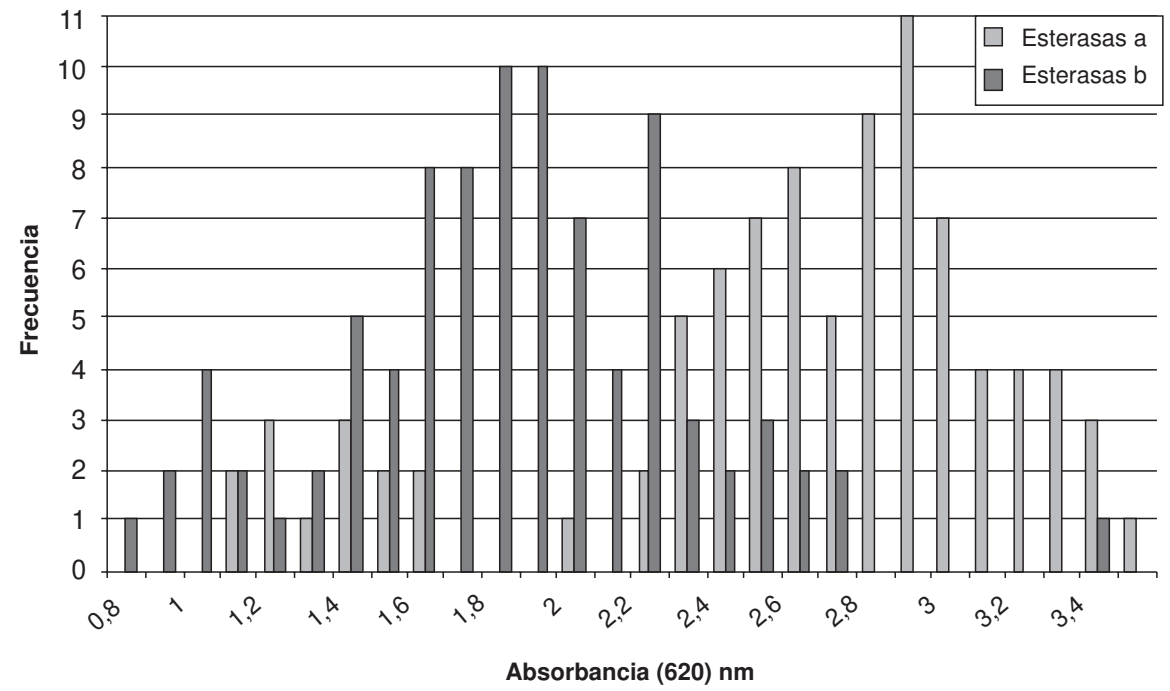

Figura 3d. Niveles de esterasas en Anopheles aquasalis de Río de Agua, municipio Libertador, estado Sucre (N=90)

$(F=28,44)$. Además, se aplicó la prueba de medias de Bonferroni y se encontró similitud entre las poblaciones de Río de Agua y Platanito, y diferencias significativas entre éstas y los mosquitos de Guayana y Catuaro.

En la figura 4 se muestran las frecuencias de los valores de oxidasas, los cuales oscilaron entre 0,1 y 2,3 . Se encontró similitud entre Catuaro y Guayana, y diferencias significativas $(p<0,0001)$ entre éstas y las de Platanito y Río de Agua $(\mathrm{F}=154,27)$.

Por otro lado, la actividad enzimática de la glutatión-S-transferasa de todas las poblaciones de mosquitos se muestra en la figura 5 ,con valores de absorbancia que oscilaron entre 0,15 y 0,23; sin embargo, sólo Río de Agua resultó positivo para este mecanismo.

En el cuadro 1 se presenta el ANOVA de una sola vía, el cual reveló diferencias significativas $(p<0,0001)$ entre las poblaciones de mosquitos $(F=72,14)$. Se aplicó la prueba de medias de Bonferroni y se encontró similitud entre las poblaciones de Guayana y Platanito; además, se encontraron diferencias significativas entre éstas y las de los mosquitos de Catuaro y Río de Agua.
En la figura 6 se muestra la frecuencia de los porcentajes de inhibición de laacetilcolinesterasa, encontrándose distintos niveles entre las poblaciones de mosquitos evaluadas. De Catuaro, $82,14 \%$ de los individuos presentaron AChe (IA); $14,28 \%$ AChe (A) y 3,57\% AChe normal. Por otra parte, en Guayana, 74,4\% de los individuos presentaron AChe (IA) y $25,6 \%$ AChe (A). Así, también, 58,9\% de los mosquitos de Río de Agua presentaron AChe (IA) y 41,1\% mostraron AChe $(A)$; en ambas cepas no se encontraron individuos con AChe normal, a diferencia de los mosquitos de Platanito, de los cuales $23,3 \%$ presentaron AChe normal, $55,6 \%$ AChe $(I A)$ y $21,1 \%$ AChe $(A)$. El ANOVA de una sola vía reveló diferencias significativas $(p<0,0001)$ entre las poblaciones de mosquitos para $A C h e$ inhibida $(F=59,72)$ y para $A C h e$ no inhibida $(F=82,36)$.

La prueba de medias de Bonferroni encontró similitud entre las poblaciones de Guayana y Río de Agua, y diferencias significativas entre éstas y los mosquitos de Catuaro y Platanito.

\section{Discusión}

El uso de insecticidas sigue siendo la estrategia de control más efectiva en la 


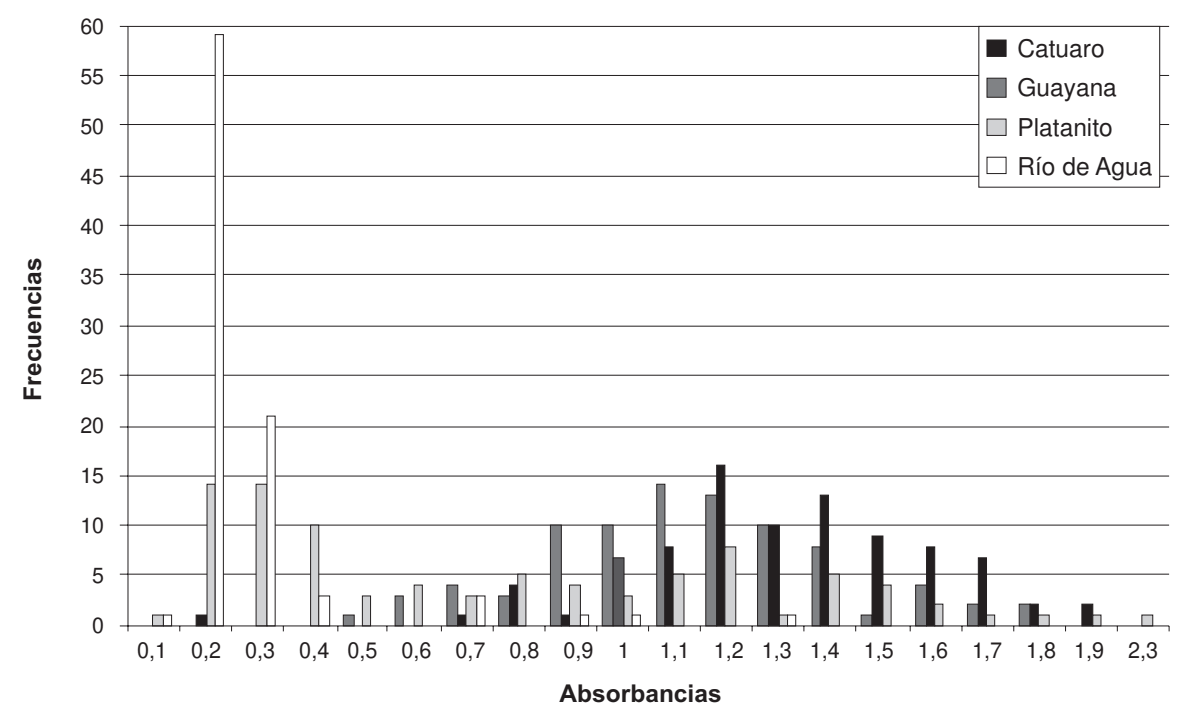

Figura 4. Frecuencia de la distribución de nivele de oxidasas obtenidos a partir del ensayo de la hemoperoxidasa en mosquitos adultos de Anopheles aquasalis, municipio Libertador, estado Sucre.

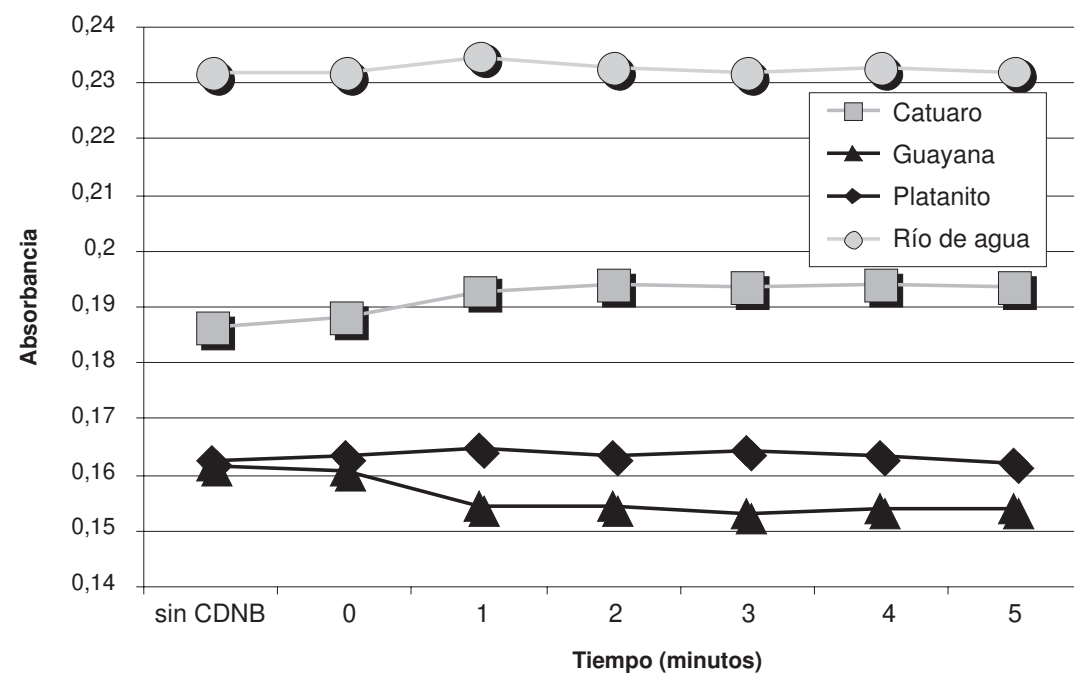

Figura 5. Actividad enzimática glutatión-S-transferasa en adultos de Anopheles aquasalis, municipio Libertador, estado Sucre.

lucha contra la malaria. Lamentablemente, el creciente desarrollo de resistencia a insecticidas amenaza esta alternativa, comprometiendo a los programas de control vectorial a implementar la vigilancia periódica de la resistencia en especies de vectores.

La combinación de datos biológicos y bioquímicos utilizada en este trabajo permitió detectar niveles de resistencia de An. aquasalis de cuatro localidades del municipio Libertador, estado Sucre, con transmisión activa de malaria, mediante diferentes mecanismos enzimáticos, a fenitrotión y a metil-pirimifos.

No obstante, los niveles de resistencia encontrados para el fenitrotión en el presente trabajo fueron mayores en una o dos veces al encontrado con metil-pirimifos, en las localidades Río de Agua, Platanito y Guayana. 


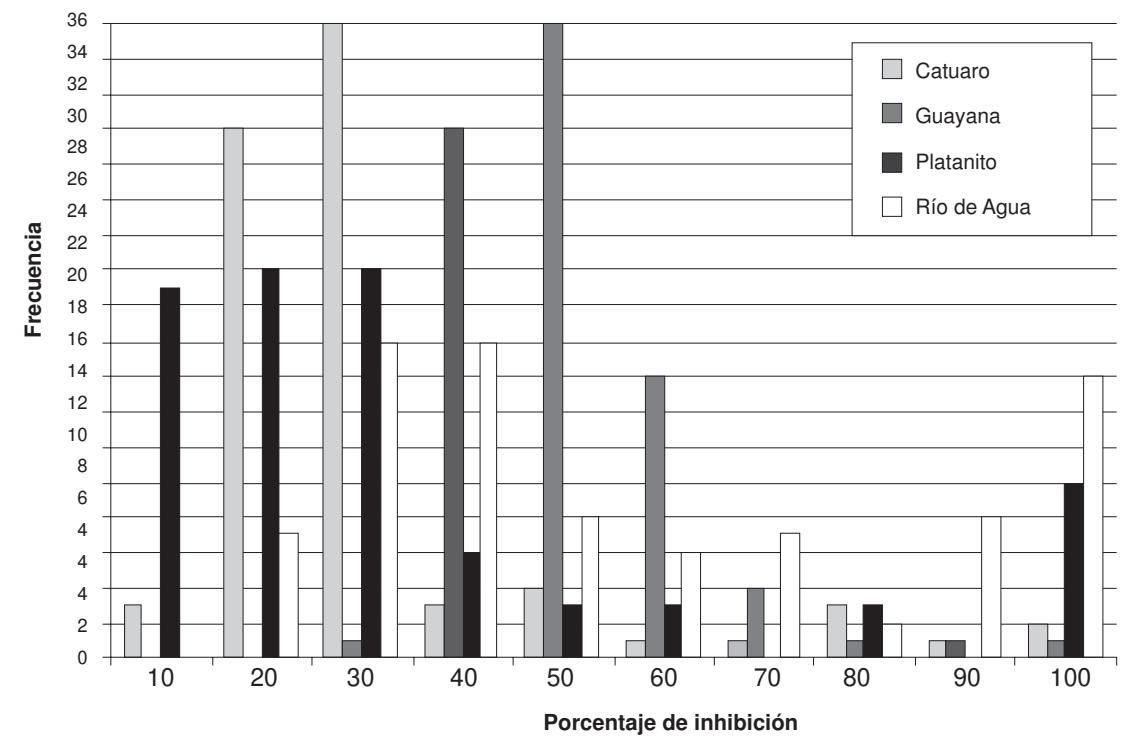

Figura 6. Porcentaje de la actividad acetilcolinesterasa inhibida con el insecticida carbamato Propoxur en adultos de Anopheles aquasalis, municipio Libertador, estado Sucre.

Esto indica que, por el uso intensivo del fenitrotión, éste ejerció presión selectiva sobre las poblaciones de mosquitos que se intentaba controlar, desarrollando en el tiempo poblaciones resistentes, a excepción de los mosquitos de Catuaro en los que el nivel de resistencia fue 1,5 veces mayor a metil-pirimifos.

Los resultados constituyen una alerta para implementar estrategias dirigidas al manejo y vigilancia de la resistencia de dichas poblaciones, dado a que el fenitrotión y el metil-pirimifos son los insecticidas organofosforados más usados por las autoridades de salud para el control de la malaria en las localidades estudiadas.

Mediante pruebas bioquímicas se demostró que las enzimas esterasas y oxidasas están actuando como mecanismos específicos de resistencia en las poblaciones de $A n$. aquasalis de las localidades evaluadas, lo que compromete a los insecticidas fenitrotión y metilpirimifos, por medio de las esterasas. Los altos valores de absorbancia para las esterasas alfa y beta caracterizan a An. aquasalis de Catuaro, Guayana, Río de Agua y Platanito como resistentes para los insecticidas mencionados.

Estos resultados concuerdan con Rodríguez et al. (19), quienes reportaron la actividad de esterasas inespecíficas y GST, para Culex quinquefasciatus y Ae. aegypti de Venezuela y Cuba. Asimismo, Flores et al. (20) reportaron que en hembras adultas de cepas de $A e$. aegypti mexicanas, de las localidades de Benito Juárez y Cozumel, se presentaron niveles elevados de esterasas alfa y beta, característicos del mecanismo de resistencia de esterasas; y ocurrieron de manera focal, con alta frecuencia en áreas turísticas donde el uso de insecticidas es intensivo. Figueroa Acosta et al. (13) identificaron en An. aquasalis de Puerta Negra, Aragua, Venezuela, el mecanismo de esterasas inespecíficas empleando el sinergista de DEF con el organofosforado metil-pirimifos; además, confirmaron elevación de las esterasas por métodos bioquímicos. Molina de Fernández et al. (21) reportaron resistencia múltiple a insecticidas en An. marajoara de Calabozo, Guárico, Venezuela, indicando aumento en el nivel de enzimas esterasas no específicas. Brogdon et al. (14) también identificaron el mecanismo de esterasas elevadas en Culex sp., el cual confiere resistencia a una amplia variedad de organofosforados.

También se han desarrollados ensayos bioquímicos para medir niveles de enzimas oxidasas en mosquitos, relacionadas con la 
resistencia a insecticidas, y se conocen valores elevados de tales enzimas en la expresión de tolerancia a insecticidas organofosforados y piretroides. Dicho mecanismo fue señalado por Brogdon et al. (14), quienes lo encontraron sólo en hembras de An. albimanus. Asimismo, Chareonviriyaphap et al. (22), usando el ensayo bioquímico, investigaron mecanismos de resistencia metabólica a insecticidas en colonias resistentes y una cepa sensible de la especie An. minimus, y midieron los niveles de esterasas no específicas monoxigenasa y GST. Los resultados indicaron aumento de la actividad de la monoxigenasa en las poblaciones seleccionadas.

No obstante, Surendran et al. (23) reportaron, en poblaciones de Phlebotomus argentipus de Egipto, que solamente en unos pocos individuos se detectó aumento de la actividad de GST y alteración de la AChe. Esto puede representar resistencia seleccionada por el rociamiento con malatión.

Por el contrario, en este estudio la actividad enzimática de la glutatión-S-transferasa no está influenciando la resistencia en $A n$. aquasalis de tres de las localidades estudiadas, a los insecticidas fenitrotión y pirimifos metil. Sin embargo, el mecanismo se presentó en mosquitos de Río de Agua, lo cual pudiera deberse a que esta actividad enzimática sea un efecto fisiológico no relacionado con el estatus de resistencia o, que dado a que la GST se encuentra asociada principalmente con resistencia a DDT, y la zona de estudio ha sido sometida a fuerte presión con insecticidas organoclorados, específicamente el DDT, ejercida por los organismos de salud para el control de la malaria (7), en concordancia con lo reportado por Penilla et al. (24), quienes demostraron que la resistencia a DDT en An. albimanus en México fue causada por niveles elevados de GST, catalizando la dehidroclorinación del DDT a su metabolito menos tóxico DDE.

Las altas frecuencias de AChe (IA) y AChe (A) detectadas en las poblaciones de An. aquasalis de Río de Agua y Guayana, indican que el fenómeno de la resistencia está presente y se está expresando con gran formas heterocigotas y también con formas homocigotas para la resistencia a fenitrotión y metil-pirimifos, mientras que los mosquitos de Catuaro y Platanito aún expresan un porcentaje de individuos con AChe normal.

Estos resultados concuerdan con los reportados con Dzul et al. (25), en los cuales las poblaciones de An. albimanus de la localidad de La Unión, México, resultaron resistentes a metil-pirimifos, con mecanismos basados en Ache. De igual manera, Zayed et al. (5) encontraron niveles significativamente altos de AChe en poblaciones de Culex pipiens de Assiut y Qalubiya, Egipto. Por otra parte, Montella et al. (19) encontraron una AChe no alterada en cepas de Ae. aegypti de Brasil. Molina de Fernández et al. (21) encontraron resistencia múltiple a insecticidas en An. marajoara de Calabozo, Guárico, Venezuela, evidenciando acetilcolinesterasa insensible. La resistencia a organofosforados fue fuertemente reducida por el sinergista butóxido de piperonilo, demostrando la participación de oxidasas de función múltiple.

La información obtenida en el presente trabajo caracteriza la resistencia en poblaciones de $A n$. aquasalis de zonas maláricas del estado Sucre, confirma la resistencia a organofosforados detectada, por lo que se piensa que dicha resistencia se debe a la presión ejercida por los servicios de salud con insecticidas durante largos periodos, el mismo tuvo un efecto selectivo produciendo un aumento en oxidasas de función múltiple, el cual puede afectar también la sensibilidad a insecticidas piretroides.

Estos resultados proveen información para la actualización de la línea de sensibilidad a insecticidas organofosforados de uso rutinario en programas de control de malaria local, así como también para la identificación de los mecanismos de resistencia que permitió determinar el espectro de resistencia, lo cual facilita la escogencia de insecticidas alternativos y permite el mapeo detallado de áreas con poblaciones resistentes.

Mientras no haya alternativas efectivas al uso del control químico, éste debe enmarcarse en 
programas de control integrado, adaptándose a las condiciones locales, con énfasis en la rotación de insecticidas, a fin de prevenir, retardar o revertir el desarrollo futuro de resistencia en las poblaciones de insectos vectores (26). La evaluación periódica de la sensibilidad de los vectores a los insecticidas, así como la investigación sobre los factores que promueven o retardan la selección de formas de resistencia, son fundamentales en la elaboración de políticas de manejo y control, en la identificación del momento oportuno y la forma correcta de realizar cambios, y la forma correcta de realizar en los tipos de insecticidas para mantener sensibles las poblaciones de vectores (27).

Por lo tanto, es esencial disponer de métodos adecuados para detectar la resistencia antes que ésta se convierta en un problema operativo. La vigilancia de los cambios en la frecuencia de los mecanismos de resistencia a insecticidas es un factor esencial para usuarlos correctamente, pues es el medio más efectivo para evitar o retardar la aparición de la resistencia a insecticidas.

El estudio realizado es de gran valor para la salud pública en Venezuela y otras regiones, como la Costa Atlántica de Colombia, donde también se encuentra este vector de la malaria. Por lo tanto, la difusión de estos resultados de resistencia a insecticidas en el vector $A n$. aquasalis es fundamental para el abordaje de la malaria en el continente americano.

\section{Agradecimientos}

Al personal del Centro de Investigaciones de Enfermedades Endémicas y Salud Ambiental y Personal del Laboratorio Entomológico de Malaria del IAESP-Ministerio del Poder Popular para la Salud.

\section{Conflicto de interés}

Los autores manifestamos que no existe conflicto de interés en el contenido de esta publicación.

\section{Financiación}

La investigación fue financiada a través del proyecto FONACIT AS-2000001898 (Ministerio de Ciencia y Tecnología), coordinado por
Darjaniva Molina, además recibió apoyo logístico del Instituto de Altos Estudios en Salud Pública "Dr. Arnoldo Gabaldon" (IAESP-Ministerio del Poder Popular para la Salud).

\section{Referencias}

1. Centers for Disease Control and prevention. Paludismo (malaria). 2007. Fecha de consulta: 5 de octubre de 2008. Disponible en:http://www2a. cdc.gov/podcasts/player.asp? $\mathrm{f}=7849$

2. Insecticide Resistance Action Committee. Prevention and management in vectors and pests of public health importance. 2006. Fecha de consulta: 6 de octubre 6 de 2008. Disponible en: http://www.irac-online.org/documents/vectormanual. pdf

3. Brogdon W, McAllister J. Insecticide resistance and vector control. Synopses. 1998;4:605-13.

4. Hemingway J, Ranson H. Insecticide resistance in insects vectors of human disease. Annu Rev Entomol. 2000;45:371-91.

5. Zayed A, Szumlas D, Hanafi H, Fryauff D, Mostafa A, Allam K, et al. Use of bioassay and microplate assay to detect and measure insecticide resistance in field populations of Culex pipiens from filariasis endemic areas of Egypt. J Am Mosq Control Assoc. 2006;22:473-82.

6. Braga I, Valle D. Aedes aegypti: insecticidas, mecanismos de ação e resistência. Epidemiol Serv Saúde. 2007;16:279-93.

7. Molina de Fernández D, Saume F, Bisset $\mathrm{J}$, Hidalgo $\mathrm{O}$, Anaya $\mathrm{W}$, González J, et al. Establecimiento de la línea de susceptibilidad de la fase adulta de Anopheles Spp. a insecticidas químicos. Bol Malariol Salud Ambient. 1997;37: 55-69.

8. Berti J, Ramírez X, González J, Herrera M. Evaluación de la efectividad de Bacillus sphaericus contra larvas de Anopheles aquasalis Curry (Diptera: Culicidae) en criaderos naturales del estado Sucre, Venezuela. Entomotropica. 2002;17:1-5.

9. Ministerio del Poder Popular para la Salud. Informe técnico para el año 2008 de la Dirección de Endemias Rurales, Carúpano, estado Sucre, Venezuela. Carúpano: Ministerio del Poder Popular para la Salud; 2008.

10. Berti J, Zimmerman R, Amarista J. Adult abundance, biting behavior and parity of Anopheles 
aquasalis Curry 1932 in two malarious areas of Sucre State, Venezuela. Mem Inst Oswaldo Cruz. 1993;88:363-9.

11. Berti J, Gutiérrez A, Zimmerman R. Relaciones entre tipos de hábitat, algunas variables químicas y la presencia de larvas de Anopheles aquasalis Curry y Anopheles pseudopunctipennis Theobald en un área costera del estado Sucre, Venezuela. Entomotropica. 2004;19:79-84.

12. Brogdon W, McAllister J. Simplification of adult mosquito bioassays through use of time mortality determinations in glass bottles. J Am Mosq Control Assoc. 1998;14:159-64.

13. Figueroa LE, Marín M, Pérez E, Molina de Fernández D. Mecanismos de resistencia a insecticidas organosintéticos en una población de Anopheles aquasalis Curry (Diptera: Culicidae) del estado Aragua. Bol Malariol Salud Ambient. 2006;46:39-47.

14. Brogdon W, Beach R, Stewart J, Castanaza L. Microplate assay analysis of the distribution of organophosphate and carbamate resistance in Guatemalan Anopheles albimanus. Bull World Health Organ. 1988;66:339-46.

15. Brogdon W, Barber M. Microplate assay of Glutathione-s-transferase activity for resistance detection in single-mosquito triturates. Comp Biochem Physiol. 1990;96:339-42.

16. Brogdon W, McAllister J, Valule J. Hemeperoxidase activity measured in single mosquitoes identifies individuals expressing an elevated oxidase for insecticide resistance. J Am Mosq Control Assoc. 1997;13:233-7.

17. Hemingway J. Techniques to detect insecticide resistance mechanisms (field and laboratory manual). Geneva: World Health Organization; 1998.

18. Flores A, Salomón J, Fernández I, Ponce G, Loaiza M, Lozano S, et al. Mechanisms of insecticide resistance in field populations of Aedes aegypti (L) from Quintana Roo, Southern Mexico. J Am Mosq Control Assoc. 2006;22:672-7.

19. Montella I, Martins A, Viana-Medeiros P, Pereira J, Braga I, Valle D. Insecticide resistance mechanisms of brazilian Aedes aegypti populations from 2001 to 2004. Am J Trop Med Hyg. 2007;77:467-77.

20. Rodríguez M, Bisset J, Molina D, Díaz C, Soca A. Adaptación de los métodos en placas de microtitulación para la cuantificación de la actividad de esterasas y glutatión-s-transferasa en Aedes aegypti. Rev Cubana Med Trop. 2001;53:32-6.

21. Molina de Fernández D, Figueroa LE, Pérez E. Resistencia múltiple a insecticidas en Anopheles marajoara Galvao \& Damasceno, 1942 en zonas agrícolas. Salud \& Desarrollo Social. 2007;3:19-29.

22. Chareonviriyaphap $\mathbf{T}$, Rongnoparut $\mathbf{P}$ Chantarumporn $\mathbf{P}$, Bangs $\mathbf{M}$. Biochemical detection of pyrethroid resistance mechanisms in Anopheles minimus in Thailand. J Vector Ecol. 2003;28:108-16.

23. Surendran S, Karunaratne S, Adams Z, Hemingway J, Hawkes $\mathbf{N}$. Molecular and biochemical characterization of a sand fly population from Sri Lanka: evidence for insecticide resistance due to altered esterases and insensitive acetylcholinesterase. Bull Entomol Res. 2005;95:371-80.

24. Penilla R, Rodríguez A, Hemingway J, Torres J, Arredondo-Jiménez J, Rodríguez M._Resistance management strategies in malaria vector mosquito control. Baseline data for a large-scale field trial against Anopheles albimanus in Mexico. Med Vet Entomol. 1998;12:217-33.

25. Dzul F, Penilla R, Rodríguez A. Susceptibilidad y mecanismos de resistencia a insecticidas en Anopheles albimanus del sur de la Península de Yucatán, México. Salud Pública Mex. 2007;49:30211.

26. Fonseca I. Estatus de la resistencia a insecticidas de los vectores primarios de malaria y dengue en Antioquia, Chocó, Norte de Santander y Putumayo, Colombia. (Tesis doctoral). Medellín: Instituto de Biología, Facultad de Ciencias Exactas y Naturales, Universidad de Antioquia; 2008.

27. Najera JA, Zaim M. Malaria vector control: Decision making criteria and procedures for judicious use of insecticides. WHO Pesticide Evaluation Scheme (WHOPES). Geneva: World Health Organization; 2002. 